\title{
Network Layer Protocols for Wireless Sensor Networks: Existing Classifications and Design Challenges
}

\author{
A. K. Dwivedi \\ School of Studies in Computer \\ Science \& I.T., \\ Pandit Ravishankar Shukla \\ University, \\ Raipur, C.G., INDIA - 492010
}

\author{
O. P. Vyas \\ Indian Institute of Information \\ Technology-Allahabad (IIIT-A), \\ Deoghat, Jhalwa, Allahabad, \\ U.P., INDIA - 211012
}

\begin{abstract}
Classifying and designing routing mechanisms or protocols for Wireless Sensor Networks are challenging due to the some inherent characteristics (energy efficiency and awareness, connection maintenance, minimum resource usage limitation, low latency, load balancing in terms of energy used by sensor nodes, etc.) that distinguish this network from the other wireless networks such as mobile ad hoc networks, cellular networks, and wireless mesh networks. Due to these unique inherent characteristics, it is a challenging task to select or propose a new algorithm for a specific WSN application. This contribution is basically a detail survey which is organized in two folds. In first fold focus is on issues on which WSN routing protocols has been categorized or classified. Second fold exploring the issues that are actually challenges which must be considered while selecting or designing an algorithm for routing purpose in WSNs.
\end{abstract}

\section{General Terms}

Algorithms, Classifications, Design Challenges.

\section{Keywords}

Wireless Sensor Networks, Routing Protocols, Protocol Classifications, Design Challenges.

\section{INTRODUCTION}

Wireless networks can be categorized as Infrastructure based (communication over an existing infrastructure, means there is a base and which gives permission to communicate) or Infrastructure less (a peer to peer communication, means there is no base and no one gives permission to communicate).

Wireless sensor nodes are tiny light weighted sensing devices consists of a constrained processing unit, little memory, EEPROM or Flash memory for tiny operating systems and other desired programs, one or more sensors, a limited range transceiver, battery or solar based power unit and optionally a mobility subsystem for mobile nodes.

Wireless Sensor Networks (WSNs) is an Ad hoc wireless networks which consists of a large number of densely deployed miniature disposable sensor nodes in the region of interest to monitor and capture physical environmental conditions (pressure, temperature, humidity, etc.) and transmit environmental data in multi hop manner to the base station/sink node for further processing through a wireless link.

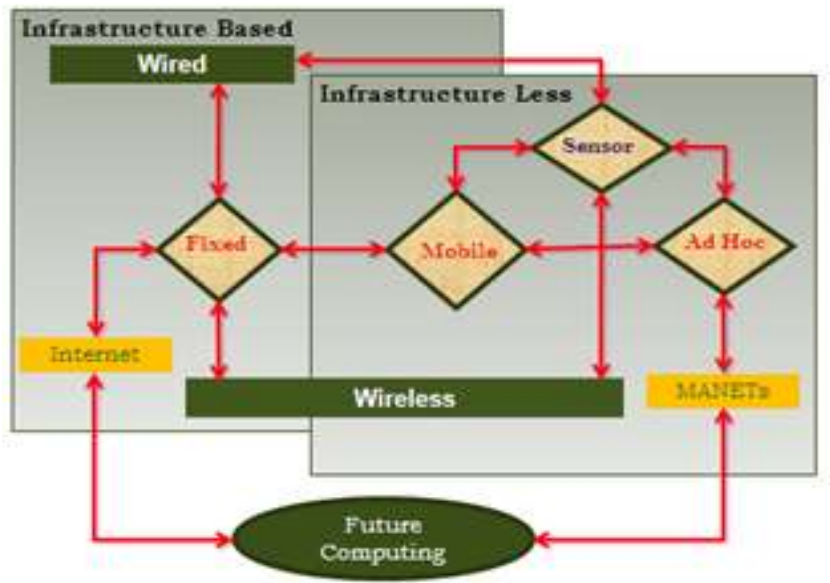

Figure 1. Types of Networks

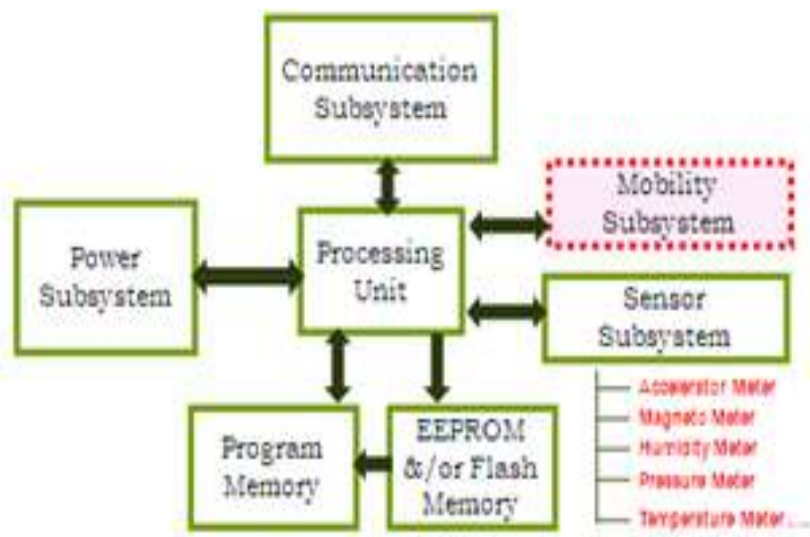

Figure 2. Wireless Sensor Node's Architecture

Wireless Sensor Network (WSN) Technology has been listed as one of the "10 Emerging Technologies That Will Change the World" by MIT Technology Review [1]. WSN is an enabling technology with the potential to revolutionize Information and Communication Technology. Applications of WSNs extend to vast and diverse areas such as the Industrial Monitoring, Building Automation, Environmental Monitoring, Disaster Response, Precision Agriculture etc. 


\section{CLASSIFICATIONS OF ROUTING PROTOCOLS FOR WIRELESS SENSOR NETWORKS}

There are many protocols proposed for WSNs but most of them were not implemented or, in the best cases, they are in a developing stage. The task of finding and maintaining routes in WSNs is nontrivial since energy restrictions and sudden changes in node status (e.g., failure) cause frequent and unpredictable topological changes. To minimize energy consumption, routing techniques proposed in the literature for WSNs employ some well-known routing tactics as well as tactics special to WSNs, e.g., data aggregation and in-network processing, clustering, different node role assignment, and data-centric methods were employed.

\subsection{Classification based on Network Structure}

Depending on the network structure, routing in WSNs can be classified into [2]:

- $\quad$ Flat-based or Data Centric routing

- Hierarchical-based or Cluster based routing

- $\quad$ Location-based routing

\subsubsection{Flat-based or Data Centric Routing}

In flat-based routing algorithm, all nodes play the same role and mainly apply flood based data transferring. The drawbacks of flooding include implosion, which is caused by duplicate messages sent to the same node, overlap when two nodes sensing the same region send similar packets to the same neighbor, and resource blindness in consuming large amounts of energy without consideration for energy constraints.

Examples of Flat-based routing algorithms or protocols are:

1. Directed Diffusion

2. Minimum Cost Forwarding Algorithm

3. Coherent/No coherent Processing

4. SPIN(Sensor Protocols for Information via Negotiation) SPIN family of protocols is made up of four protocols:

$$
\begin{array}{ll}
\text { 1. } & \mathrm{SPIN}-\mathrm{PP} \\
\text { 2. } & \mathrm{SPIN}-\mathrm{EC} \\
\text { 3. } & \mathrm{SPIN}-\mathrm{BC} \\
\text { 4. } & \mathrm{SPIN}-\mathrm{RL}
\end{array}
$$

5. Rumor Routing

6. SER (Stream Enable Routing)

7. GBR (Gradient-Based Routing)

8. CADR (Constrained Anisotropic Diffusion Routing)

9. COUGAR
10. ACQUIRE (Active Query Forwarding in Sensor Networks)

11. TEEN (Threshold sensitive Energy Efficient sensor Network protocol) and APTEEN (Adaptive Periodic TEEN) etc.

\subsubsection{Hierarchical-based or Cluster based Routing}

Hierarchical protocols aim at clustering the nodes so that cluster heads can do some aggregation and reduction of data in order to save energy. Hierarchical routing is mainly two-layer routing where one layer is used to select cluster heads and other for routing. Nodes in hierarchical networks play different roles. Higher-energy nodes can be used to process and send the information; low-energy nodes can be used to perform the sensing in the proximity of the target. Hierarchical routing is utilized to perform energy-efficient routing in WSNs. Hierarchical routing is an efficient way to lower energy consumption within a cluster.

Examples of Hierarchical-based routing algorithms or protocols are:

1. SHRP (Simple Hierarchical Routing Protocol)

2. LEACH (Low energy Adaptive Cluster Hierarchy) routing protocol.

3. LEACH-C (LEACH Centralized) routing protocol.

4. PEGASIS (Power Efficient Gathering in Sensor Information System) routing protocol.

5. Hierarchical PEGASIS

6. HEAP (Hierarchical Energy Aware Protocol for routing and Aggregation in Sensor networks)

7. HPEQ (Hierarchical Periodic, Event-driven and Query-based)

8. TEEN (Threshold sensitive Energy Efficient sensor Network protocol) and APTEEN (Adaptive TEEN)

9. $\quad \mathrm{SMECN}$ (Small MECN)

10. SOP (Self-Organizing Protocol)

11. GAF (Geographic Adaptive Fidelity)

12. SPAN etc.

\subsubsection{Location-based Routing}

Location-based protocols utilize the position information to relay the data to the desired regions rather than the whole network. Most of the routing protocols for sensor networks require location information for sensor nodes. In most cases location information is needed in order to calculate the distance between two particular nodes so that energy consumption can be estimated. Since, there is no addressing scheme for sensor networks like IP-addresses and they are spatially deployed on a region.

Examples of Location-based routing algorithm/protocols are: 
1. MECN (Minimum Energy Communication Network) and SMECN (Small MECN)

2. GAF (Geographic Adaptive Fidelity)

3. GEAR (Geographic and Energy Aware Routing) etc.

\subsection{Classification based on Protocol Operation}

Depending on the protocol operation, routing in WSNs can be divided into [2]:

- Multipath-based routing

- Query-based routing

- Negotiation-based routing

- QoS-based routing

- Non-coherent \& Coherent data-processing based routing

\subsubsection{Multipath-Based Routing}

This type of routing protocols uses multiple paths instead of a single path in order to enhance network performance. Directed diffusion is a good example of this type of routing.

\subsubsection{Query-Based Routing}

In this type of routing protocol destination nodes propagate a query for data (sensing task) from a node through the network, and a node with this data sends the data that matches the query back to the node that initiated the query. Directed diffusion, Rumor, ACQUIRE, COUGAR are good examples of this type of routing protocol.

\subsubsection{Negotiation-Based Routing Protocols}

These protocols use high-level data descriptors in order to eliminate redundant data transmissions through negotiation. Communication decisions are also made based on the resources available to them. SPIN family protocols are good examples of negotiation-based routing protocols.

\subsubsection{QoS-Based Routing}

In QoS-based routing protocols, the network has to balance between energy consumption and data quality. In particular, the network has to satisfy certain QoS metrics (delay, energy, bandwidth, etc.) when delivering data to the base station. SPEED (Stateless Protocol for Real-Time Communication in Sensor Networks) is good example of this type of protocols.

\subsubsection{Non-coherent and Coherent Data-Processing Based Routing}

In non-coherent data processing routing, nodes will locally process the raw data before it is sent to other nodes for further processing. The nodes that perform further processing are called aggregators. Non-coherent functions have fairly low data traffic loading. In coherent routing, the data is forwarded to aggregators after minimum processing. The minimum processing typically includes tasks like time-stamping and duplicate suppression. To perform energy-efficient routing, coherent processing is normally selected. Since coherent processing generates long data streams, energy efficiency must be achieved by path optimality.

Single Winner Algorithm (SWE) is a good example of noncoherent while Multiple Winner Algorithm (MWE), a little variant of SWE is a coherent processing algorithm.

\subsection{Classification based on Packet Destination}

Recently, a new classification classifies the routing techniques according to packet destination (a single node, a set of nodes or every node in network) [3]:

- $\quad$ Gossiping and agent-based unicast forwarding

- Energy-efficient unicast

- Broadcast and multicast

- Geographic routing

- $\quad$ Mobile nodes

\subsubsection{Gossiping and agent-based unicast forwarding}

These schemas are an attempt of working without routing tables in order to minimize the overflow needed to build the tables, as much as result of the initial stages in which the tables were not built yet. The simplest choice is flooding (forwarding each message received), but it is not very efficient. Gossiping avoids the problem of implosion by selecting a random node to which to send the packet rather than broadcasting the packet blindly. However, this causes delays in propagation of data through the nodes.

\subsubsection{Energy-efficient unicast}

These techniques analyze the network nodes distribution to set the cost of transmitting over the link between two nodes and select an algorithm to calculate the minimum cost.

There are many aspects to consider about the energy awareness:

- Minimize energy per packet

- Maximize network's lifetime

- Set routes according to the remaining energy

- Minimize the amount of transmission power

\subsubsection{Broadcast and multicast}

Earlier protocols, gossiping and unicast, try to find efficient ways to send data between nodes, possibly over several hops. For this, many nodes must collect or distribute the information to every node in the network (broadcast). In fact, broadcast is a common operation in WSN applications. In a similar way, sometimes is necessary to distribute data to a subset of previously known nodes. This process is called multicast.

\subsubsection{Geographic Routing}

This kind of routing appeared due to two main motivations:

a. Many applications need the node location as a reference address to allow destinations of the type: "every node in a given region" or "the closer node to a point". If these 
requirements are needed, an appropriated routing scheme must be provided. This first idea, sending data randomly to every node in a given region is called geo-casting.

b. When the source and destination position is known and also the nodes among them, this information can be used to improve the routing process. For that, the destination node location must be specified geographically or relatively (with a location service). This second is called position-based routing.

\subsubsection{Mobile nodes}

These aspects with motion ability should be considered for wireless sensor networks: mobile sensor nodes, mobile base station, mobile sensed phenomenon or combination of these.

\subsection{Crossbow (Xbow) Routing Classification}

As discussed earlier there are many protocols proposed for WSN but most of them were not implemented or, in the best cases, they are in a developing stage. For these reasons it has been carried out a tour over the routing techniques already implemented in TinyOS. Many proposals have been found along the different TinyOS contributions, but most of them are similar. The best way of our review is to focus on one of the contributions that gave us the most number of choices that is Crossbow (Xbow) contribution. Crossbow Technology provides leadingedge Solutions in WSN Technology and is the Largest Manufacturer of WSN [4].

Crossbow (Xbow) contribution offers four kinds of routing [4]:

- Basic Routing (with normal or improved variants)

- Reliable Routing

- $\quad$ Low Power Routing

- XMesh Routing

\subsection{Classification based on State}

Another type of classification is as follows [5]:

- $\quad$ Stateful Ad Hoc Routing

- $\quad$ Stateless Geometric Ad Hoc Routing

\subsubsection{Stateful Ad Hoc Routing}

The stateful ad hoc routing protocols require node to maintain some routing information that is collected using the routing protocol (e.g., through route request propagation or by reversing paths taken by the query). Stateful routing protocols need the routing information maintained at each intermediate node through the data forwarding path. More specifically, state is kept at some nodes about non-local areas in the network (for example, the path to reach some non-local node).

Examples of Stateful Ad Hoc routing algorithm/protocols are:

1. Dynamic Source Routing (DSR)

2. AODV

3. DSDV

\subsubsection{Stateless Geometric Ad Hoc Routing}

These kinds of protocols only track the position of their neighbors and select among them a neighbor that is likely to be closer to the destination. Stateful routing may not be efficient or even possible for very large networks with limited sensor node capabilities. Accordingly, stateless routing protocols which do not maintain per-route state have been proposed. They scale effectively in terms of routing overhead because the tracked routing information does not grow with the network size or the number of active sinks. Geographic (and more generally location based) routing protocols are the main type of stateless routing protocols.

Examples of Stateless Geometric Ad Hoc routing algorithm/protocols are:
1. Greedy/Geographic Forwarding
2. Face Routing
3. GPSR
4. COMPASS

\subsection{Classification based on Epidemic behavior}

Another classification of wireless sensor network routing protocol is available in a research contribution [6]. Here routing protocol follow the model of nature to spread information and define simple rules for information to flow between nodes of a network. Epidemic algorithms can be differentiated from each other by their style of communication between neighboring nodes:

- Pull based epidemic algorithm

- $\quad$ Push based epidemic algorithm

- $\quad$ Pull-push based epidemic algorithm

\subsubsection{Pull based epidemic algorithm}

A node asks a selected neighbor for new information. The node will receive new information only if the neighbor has new information.

\subsubsection{Push based epidemic algorithm}

A node with new information sends the information to a selected neighbor.

\subsubsection{Pull-push based epidemic algorithm}

This algorithm is a combination of two models described above. A node employing such an algorithm sends information to a selected neighbor when it has some information available; it also asks and receives new information from the selected neighbor if the neighbor has new information.

In another classification routing protocols is broken down based on following techniques:

- $\quad$ Flooding

- Gradient

- $\quad$ Clustering 


\section{- Geographic}

Apart from these classifications routing protocols can be classified on the basis of whether they have security mechanism or not, whether they are energy efficient or not, whether they are for real-time applications or not, etc.

\section{DESIGN CHALLENGES}

Routing in WSNs is very challenging due to unique inherent characteristics (energy efficiency and awareness, connection maintenance, minimum resource usage limitation, low latency, load balancing in terms of energy used by sensor nodes, etc.) that distinguish this network from other wireless networks such as mobile ad hoc networks, cellular networks, and wireless mesh networks. Major Constraints while designing protocols for WSNs are: Energy, Processing power, Memory. In various literatures or research contributions, related to WSNs these design challenges are identified $[2,3,5,7,8,9,10]$ :

1. Due to the relatively large number of sensor nodes, it is not possible to build a global addressing scheme for the deployment of a large number of sensor nodes as the overhead of ID maintenance is high. Thus, traditional IPbased protocols may not be applied to WSNs.

2. In contrast to typical communication networks, almost all applications of sensor networks require the flow of sensed data from multiple sources to a particular Base Station.

3. Sensor nodes are tightly constrained in terms of energy, processing, and storage capacities. Thus, they require careful resource management.

4. In most application scenarios, nodes in WSNs are generally stationary after deployment except for, may be, a few mobile nodes.

5. Sensor networks are application specific, i.e., design requirements of a sensor network change with application.

6. Position awareness of sensor nodes is important since data collection is normally based on the location.

7. Finally, data collected by various sensors in WSNs is typically based on common phenomena; hence there is a high probability that this data has some redundancy.

Visibility [10] is a new metric for WSNs protocol design. The objective of this visibility metric is that "Minimize the energy cost of diagnosing the cause of a failure or behavior".

These are the some parameters on which routing protocols must be evaluated during designing new one: Classification/Category, Power Usage, Data Aggregation, Scalability, Query-based, Latency (delay), Overhead, Data Delivery Model, Quality of Service, Security.

Except above these parameters these areas should also need attention while evaluating routing protocols for WSNs:

- $\quad$ Node Deployment option

- Topology
- Sensor Density or Network Size

- Environment or Scenario

\section{CONCLUSION}

This research contribution is basically a detail survey which is organized into two folds. In first fold focus is on issues on which WSN routing protocols has been categorized or classified. Second fold exploring the issues that are actually challenges which must be considered while selecting or designing an algorithm for routing purpose in WSNs. The study has clearly brought forth important findings that are very useful and presents enough valuable contents related to wireless sensor network protocol design issues and existing classifications.

\section{REFERENCES}

[1] Technology Review, Feb. 2003. www.technologyreview.com

[2] Al-Karaki, J.N. and Kamal, A.E. 2004. Routing Techniques in Wireles Sensor Networks: A Survey, IEEE Wireless Communications (J) (Dec 2004). 06-28.

[3] Karl, H. and Willig, A. 2006. Protocols and Architectures for Wireless Sensor Networks, Editorial John Wiley \& Sons Ltd., ISBN 13978-0-470-09510-2.

[4] Olivares, T., Tirado, P.J., Royo, F., Castillo, J.C. and Orozoco-Barbosa, L. 2007. IntellBuilding: A Wireless Sensor Network for Intelligent Buldings. Poster. Fourth European Conference on Wireless Sensor networks (EWSN 2007), Parallel and Distributed Systems Report Series, report number PDS-2007-00. ISSN: 1387-2109. Delft (Ned).

[5] Eriksson, J. 2009. Detailed Simulation of Heterogeneous Wireless Sensor Networks, Dissertation for the degree of Licentiate of Philosophy in Computer Science at Uppsala University, Sweden, (April 2009), ISSN 1404-5117.

[6] Akdere, M., Bilgin, C.C., Gerdaneri, O., Korpeoglu, I., Ulusoy, Ö. and Cetintemel, U. 2006. A Comparison of Epidemic Algorithms in Wireless Sensor Networks, Computer Communications (Elsevier) (J), Vol. 29, 2450 2457.

[7] Akyildiz, I.F., Su, W., Sankarasubramaniam, Y. and Cayirci, E. 2002. Wireless Sensor Networks: A Survey, Computer Networks (Elsevier) (J), Vol. 38 (March 2002), 393-422.

[8] Akkaya, K, and Younis M. 2005. A Survey on Routing Protocols for Wireless Sensor Networks, Ad Hoc Network (Elsevier) (J), Vol. 3, 325-349.

[9] Dwivedi, A.K., Tiwari, M.K. and Vyas, O.P. 2009. Operating System for Tiny Networked Sensors: A Survey, International Journal of Recent Trends in Engineering (Computer Science), Vol. 1, No. 2, 152-157.

[10] Wachs, M., Choi, Jung, J. Il, Lee, W., Srinivasan, K., Chen, Z., Jain, M., and Levis, P. 2007. Visibility: A New Metric for Protocol Design. In Proceedings of the ACM SenSys. 